\title{
Biomechanical Analysis of Hyoid Bone Displacement in Videofluoroscopy: A Systematic Review of Intervention Effects
}

\author{
Jolien G. J. van der Kruis • Laura W. J. Baijens • \\ Renée Speyer · Iris Zwijnenberg
}

Received: 10 June 2010/ Accepted: 25 November 2010/Published online: 17 December 2010

(C) The Author(s) 2010. This article is published with open access at Springerlink.com

\begin{abstract}
This systematic review explores studies using biomechanical analysis of hyoid bone displacement in videofluoroscopy of swallowing as a spatial outcome parameter to evaluate intervention effects. Two authors independently carried out the literature search using the electronic databases Embase, PubMed, and Cochrane Library. Differences in their search findings were settled by discussion. The search was limited to publications in the English, German, French, Spanish, or Dutch language. MeSH terms were used, supplemented by free-text words to identify the most recent publications. In addition, reference lists were searched by hand. Only studies using videofluoroscopy to evaluate the biomechanical effects of swallowing interventions in dysphagic subjects were included in the review. While the body of literature on measuring hyoid bone displacement in videofluoroscopy has grown, only 12 studies met the inclusion criteria. Several of the 12 studies had methodological shortcomings. In general, the conclusions could not be compared across the studies because of their heterogeneous designs and outcome measures. Overall, several intervention effect studies reported significant results. In particular, bolus modification and swallowing maneuvers showed a greater range of hyoid bone
\end{abstract}

J. G. J. van der Kruis · L. W. J. Baijens $(\bowtie) \cdot$ R. Speyer Department of Otorhinolaryngology, Head and Neck Surgery, Maastricht University Medical Center, P.O. Box 5800, 6202 AZ Maastricht, The Netherlands

e-mail: laura.baijens@mumc.nl

R. Speyer

Comprehensive Cancer Center West, Leiden, The Netherlands

I. Zwijnenberg

Department of Surgery, St. Elisabeth Hospital, Tilburg,

The Netherlands displacement. In light of this review, further research on hyoid bone displacement as a spatial variable in welldefined patient populations using well-defined videofluoroscopic protocols to measure intervention effects is recommended.

Keywords Systematic review - Hyoid bone · Biomechanical analysis · Videofluoroscopy · Deglutition · Deglutition disorders

Oropharyngeal dysphagia is a common symptom among aging individuals $[1,2]$. Swallowing disorders are also found in patients with central nervous system diseases, neuromuscular disorders, or head and neck cancers [3, 4]. Complications caused by dysphagia can have far-reaching consequences such as aspiration pneumonia, malnutrition, dehydration, or death $[5,6]$. Therefore, evaluation of oropharyngeal dysphagia by more objective methods is essential to determine which intervention is most suitable for each patient.

Videofluoroscopy (VFS) has become the accepted standard for the evaluation of swallowing [7-10]. All VFS studies include image sequences that can be digitized and analyzed using various software applications. These techniques make measurements more precise by allowing frame-by-frame analysis, thereby increasing intra- and interrater reliability $[7,11]$. Spatial, temporal, and visuoperceptual data can be collected from these studies. Several standardized procedures are used to analyze VFS studies: for example, a standardized VFS recording protocol [10]; a well-designed software application using a standard plane to correct for head postures; calibration to correct for the magnification; and several well-defined anatomic reference points of interest. Over the past 25 years, a range of 
software applications and standard planes have been described. Logemann et al. [12] presented one of the first interactive software applications to digitize, enhance, and plot the movement of anatomical structures during swallowing. In this program, VFS analysis is based on four major steps: digitization, identification of reference points and anatomical points of interest, calculation of the coordinates, and generation of spatial position plots. One of the seven anatomical points of interest is the anterior/superior corner of the hyoid bone [12]. Furthermore, several other studies have been performed to validate and improve VFS analysis [7, 9, 11, 13].

Normally, the onset of hyoid bone displacement initiates the pharyngeal phase of swallowing. This displacement is caused by contraction of the suprahyoidal muscles. Suprahyoidal muscle contraction initiates superior laryngeal movement, producing anterior traction on the cricoid. This traction results in the opening of the upper esophageal sphincter (UES) [14]. A diminished hyoid bone displacement indicates impairment of swallowing. Some consequences could be impaired bolus transport, aspiration, and abnormal opening of the UES [15]. Almost all methodological articles evaluating VFS adopt the hyoid bone as an anatomical point of interest to analyze swallowing [7, 11, 12].

Biomechanical analysis of the hyoid bone in VFS is influenced by various factors. Bolus characteristics [16], etiology of dysphagia [17], and age and gender [1, 2] have been shown to interact with swallowing. A well-designed study has to treat these as important factors.

The aim of this review article was to summarize all published articles on the spatial outcome parameter biomechanical analysis of hyoid bone displacement in VFS, used by the authors to evaluate intervention effects in dysphagic subjects. So far, no reviews have been published on this topic.

\section{Methods}

Two authors independently carried out a literature search using the electronic biomedical databases Embase, PubMed, and Cochrane Library. Differences in their search findings were settled by discussion. The search was limited to publications in English, German, French, Spanish, or Dutch. In PubMed, the MeSH term hyoid bone was combined with the terms movement or biomechanics. Next, the $\mathrm{MeSH}$ term hyoid bone was combined with deglutition or deglutition disorders. Furthermore, the MeSH terms deglutition or deglutition disorders were linked with the term biomechanics. In Embase, the thesaurus terms dysphagia or swallowing were combined with hyoid bone. Second, the thesaurus terms biomechanics or movement were combined with hyoid bone. Third, the thesaurus term biomechanics was combined with dysphagia or swallowing. To identify the most recent publications, the search was expanded by using free-text words (truncation or wildcard) for the period after September 2007 until March 2010. In PubMed, the free-text word hyoid* was combined with deglut* or swallow* or dysphag*. Next, the word hyoid $^{*}$ was combined with movement* or biomechanic*. In Embase, keywords were used in advanced ovid search. The word hyoid* was combined with biomechanic* or move$m e n t^{*}$. The word hyoid* was linked with the words swallow* or deglut* or dysphag*. In the Cochrane Library, hyoid bone was recognized as a thesaurus term and hyoid $*$ was also explored as a free-text word.

The reference lists of all the included articles were searched for additional literature. This search did not yield additional studies. Intervention studies describing videofluoroscopic data of hyoid bone displacement were included. Only articles presenting both pre- and post-intervention data of the oropharyngeal swallowing function in dysphagic subjects were included. Studies presenting data on the effect of different bolus volumes and consistencies on hyoid bone displacement were included. Articles describing dysphagia as a side effect of therapy were excluded; for example, the review excluded studies that compared swallowing in pre- and postsurgical treatment for head and neck cancer [18-22]. Review articles and studies with a subject population smaller than five were excluded [23, 24], as were experiments on animals [25]. Also excluded were studies based solely on temporal variables - thereby leaving out spatial variables as outcome parameters of hyoid bone displacement, like moment of onset of superior hyoid bone displacement [26, 27] - and articles that analyzed only qualitative measurements such as reduced hyoid bone displacement and articles that measured hyoid bone displacement by methods other than VFS [28, 29].

Both reviewers independently based their first selection on abstracts. The original articles were used to make the definitive decision on inclusion. To determine the level of evidence of the included articles, the $\mathrm{ABC}$ rating scale developed by Siwek et al. was used [30]. Level A refers to high-quality randomized controlled trials and level $\mathrm{B}$ refers to well-designed nonrandomized clinical trials. Level $\mathrm{C}$ articles presenting a consensus or an expert opinion were excluded from this study.

\section{Results}

A total of 772 articles were found. Using MeSH terms, 416 articles were selected in PubMed. Using thesaurus terms, 252 articles were found in Embase and 11 in the Cochrane 
Library. Then, a search using free-text words resulted in 37 articles from PubMed, 30 from Embase, and 26 from the Cochrane Library. Due to overlap, 177 articles were excluded, leaving 595 articles in all. Eventually, only 12 of these 595 articles met the inclusion criteria. While other well-designed studies on the biomechanical analysis of hyoid bone displacement in videofluoroscopy have been published, they do not specifically evaluate the intervention effects and could thus not be included in this review.

Table 1 (intervention effect studies) gives an overview of the included studies. The table divides the interventions into five groups: swallow postures, maneuvers, and rehabilitation exercises [31-33]; facilitation techniques [34]; bolus modification [35-37]; a combination of the mentioned interventions [38-41]; and other interventions [42].

The first column presents the level of evidence using the $\mathrm{ABC}$ rating scale according to Siwek et al. [30]. References in the second column are listed according to level of evidence and alphabetic order of the authors for the five intervention groups. The third column gives information about the number, diagnosis, and gender of the subjects. The number of patients included and covered by the $N$ in the present review refers to the patients for whom statistical analysis was applied, thus excluding the dropouts. Information about the raters is based on the number, reliability, and blinding of the raters for the spatial analyses of the hyoid bone in VFS. The other columns summarize the following data (if present in the article): interventions and treatment groups; information on software applications for biomechanical analyses; definition of the hyoid bone reference points; applied bolus size and consistency; other measurements or evaluation tool(s); and author(s)' key findings.

\section{General Results}

The number of subjects in the included studies varied from 5 (studies having less than five subjects were excluded) to 65. Most intervention effect studies analyzed rather small dysphagic populations. All studies used both descriptive statistics and statistical analysis to evaluate hyoid bone displacement. Three of the 12 studies measured intervention effects using more than one assessment tool. Five of the 12 used several landmarks on the cervical spine as reference points to analyze hyoid bone displacement in VFS, whereas six used landmarks on the hyoid bone. In most studies, a point on the anterior corner of the hyoid bone was marked for evaluation of hyoid bone displacement. All studies applied calibration by using a reference marker to correct for magnification in VFS. For calibration most investigators taped a radiopaque object to the subjects, affixing it in the field of imaging. Below, all 12 included studies are summarized briefly.
Some frequently applied rehabilitation techniques for dysphagia are swallowing maneuvers, particularly the supraglottic swallow, the super-supraglottic swallow, the effortful swallow, and the Mendelsohn maneuver. The supraglottic swallow maneuver was designed to close the airway at the true vocal folds before and during the swallow, thus preventing aspiration during the swallow (breathhold maneuver). The super-supraglottic swallow comprises the same steps as the supraglottic swallow, but during the voluntary breath-hold period, the patient is asked to bear down, increasing the effort of closure. The increased effort during the breath-hold tilts the arytenoids further forward, toward the base of the epiglottis, and pulls in the false vocal folds, generally completely closing the airway entrance above the true vocal folds. During effortful swallow, the patient has to squeeze hard with the muscles of the tongue to increase the tongue base movements during swallowing. The Mendelsohn maneuver involves swallowing normally and as the larynx elevates, catching it with neck muscles, and holding it at maximum elevation for a count of six while swallowing to improve the UES opening [43].

Rehabilitation exercises can include muscle strength training like tongue exercises and the Shaker exercise program. In the study of Shaker et al. [31], the effect of the Shaker exercise program was compared to sham exercise in dysphagic subjects in a randomized controlled trial design $(N=27)$. The Shaker exercise program was developed to strengthen the suprahyoid muscles in 6 weeks using a head-raising exercise program. Patients initially included in the sham exercise group were crossed over to perform the real exercise program for 6 weeks. Following 6 weeks of exercise, the anterior and superior hyoid bone excursion did not show significant group differences. Nevertheless, other biomechanical parameters, like anteroposterior diameter of the UES opening and maximal anterior laryngeal excursion, did increase significantly after treatment, as did the Functional Outcome Assessment Measure of Swallowing (FOAMS). Regardless of heterogeneous etiologies and duration of the dysphagic complaints, all parameters improved significantly. After completion of the Shaker exercise program, the postdeglutitive aspiration resolved and all patients were able to discontinue tube feeding. However, the predeglutitive aspiration remained. The authors concluded that the proposed suprahyoid muscle-strengthening exercise program is effective in restoring oral feeding in some patients with deglutitive failure because of abnormal UES opening.

The influence of three swallowing techniques on swallowing was analyzed by Bülow et al. [32]. Eight subjects with pharyngeal dysfunction were evaluated in a singlesession design. Three different swallowing techniques were used: chin tuck, effortful, and supraglottic swallow 


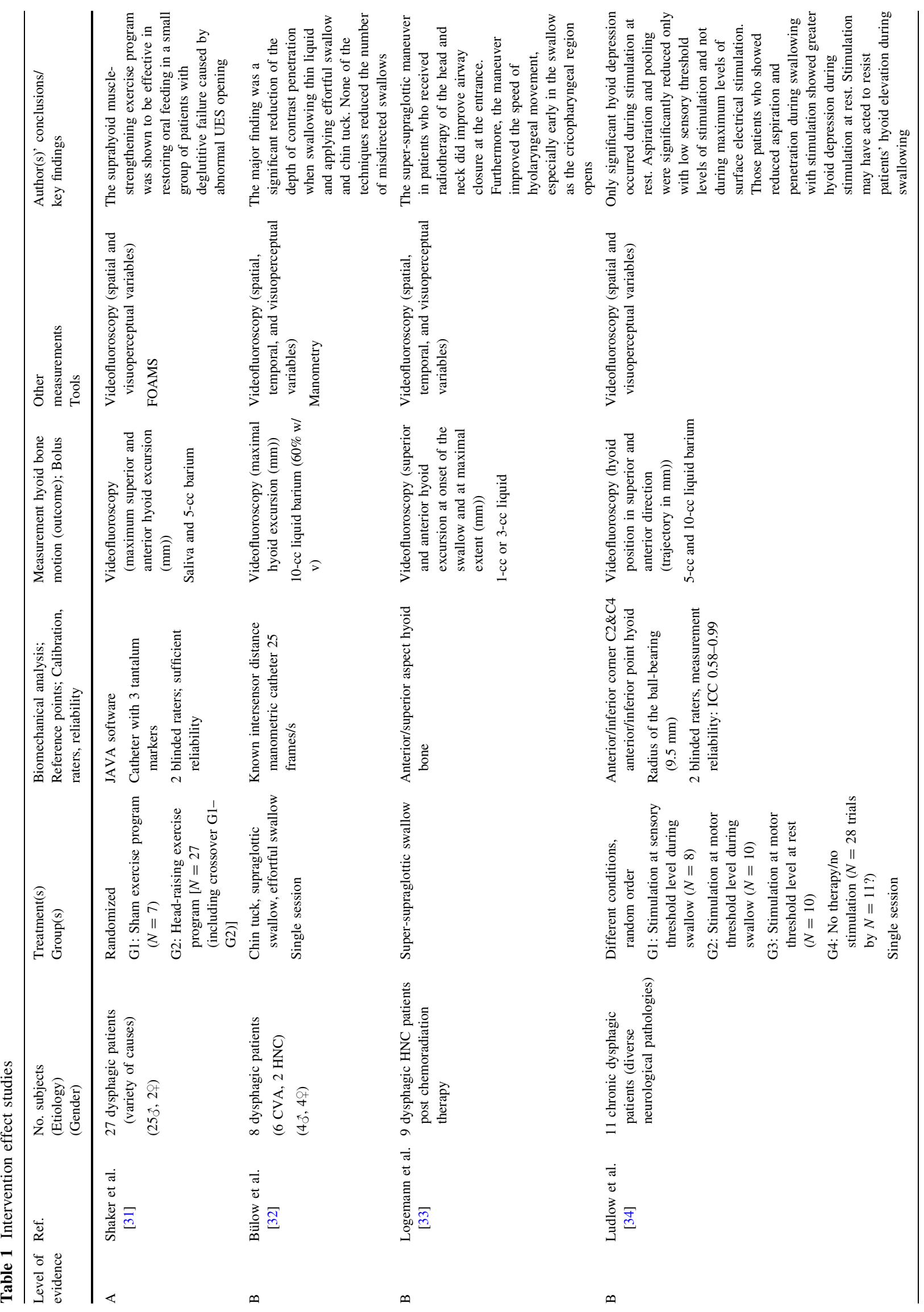




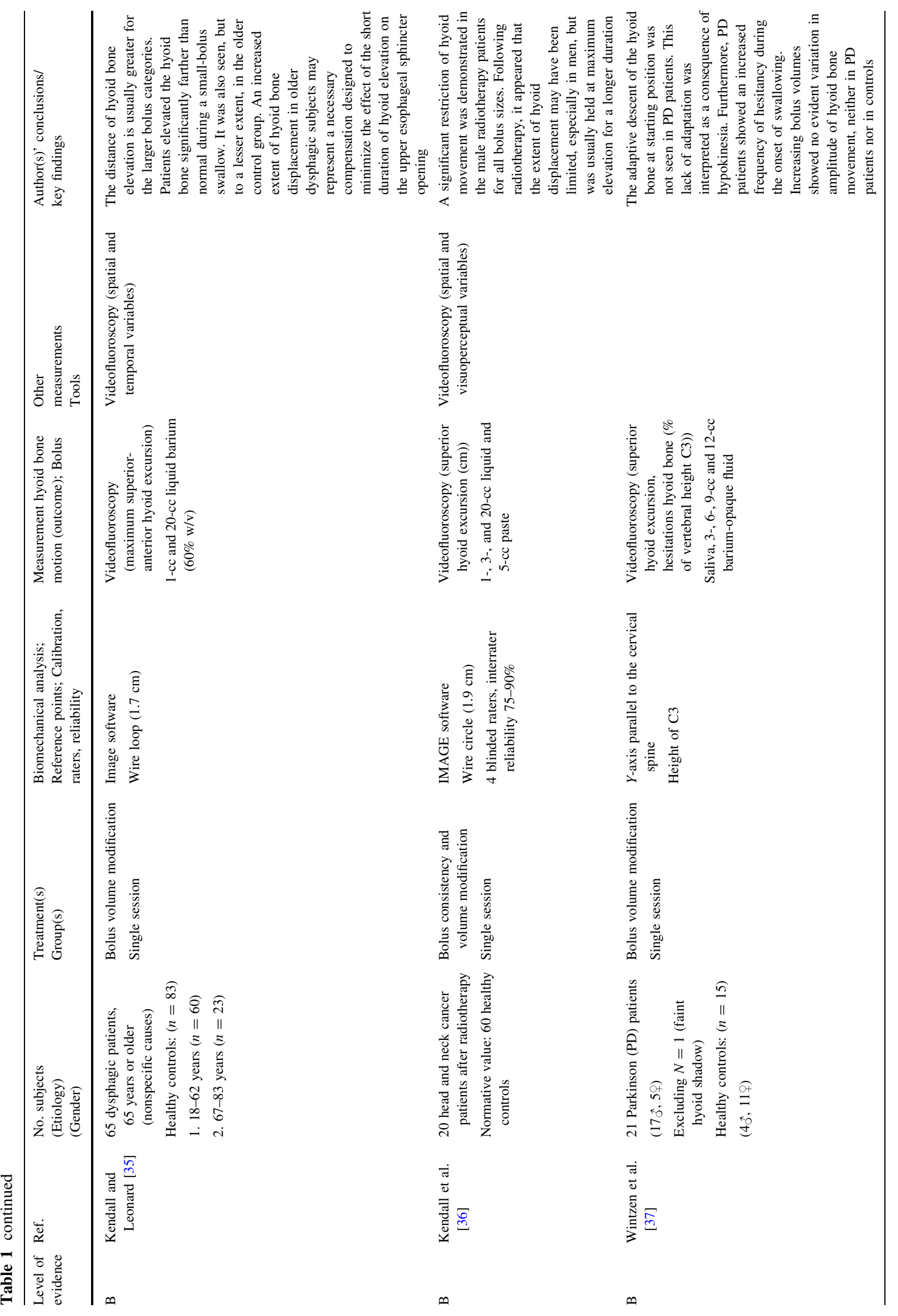




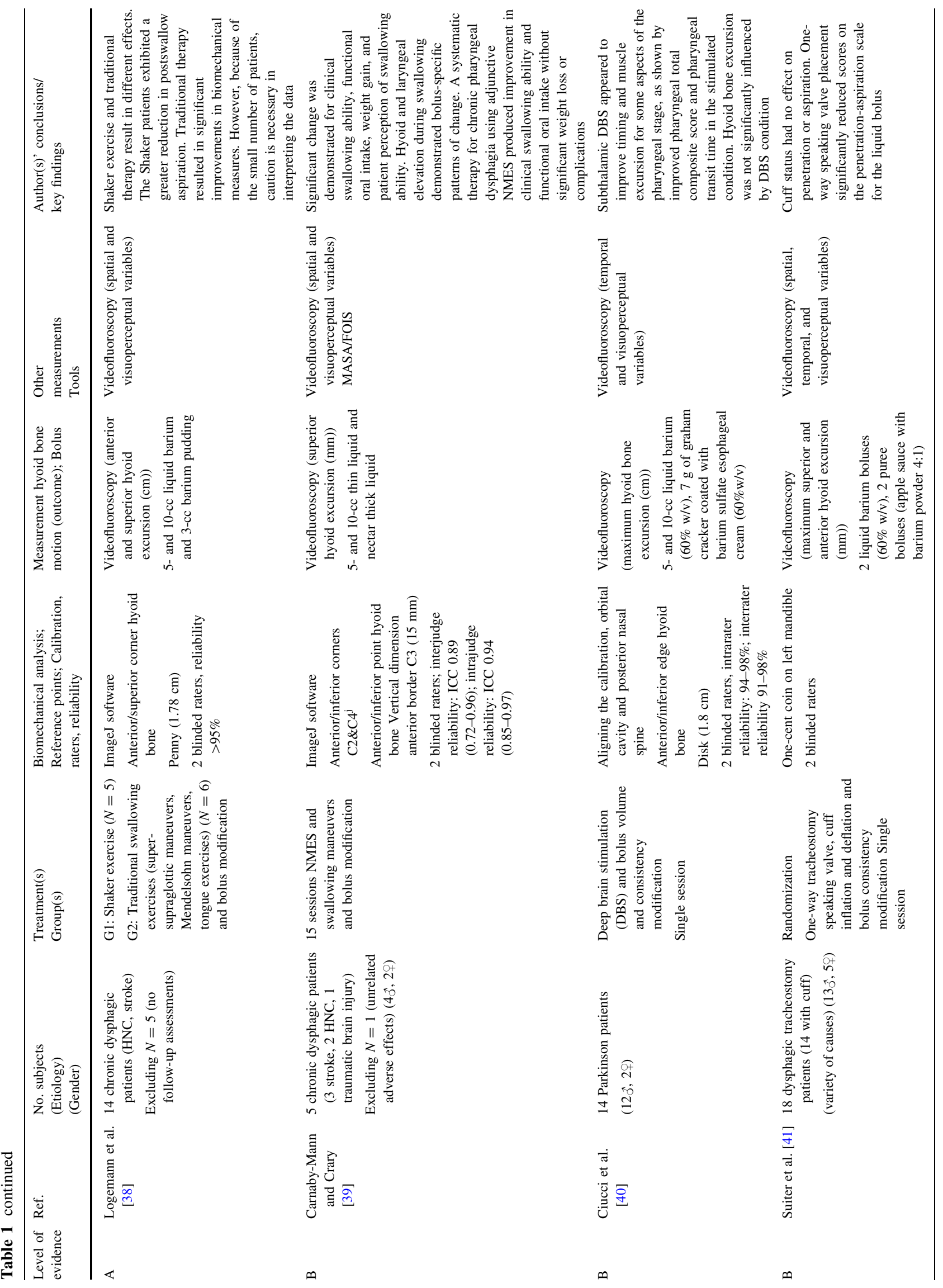




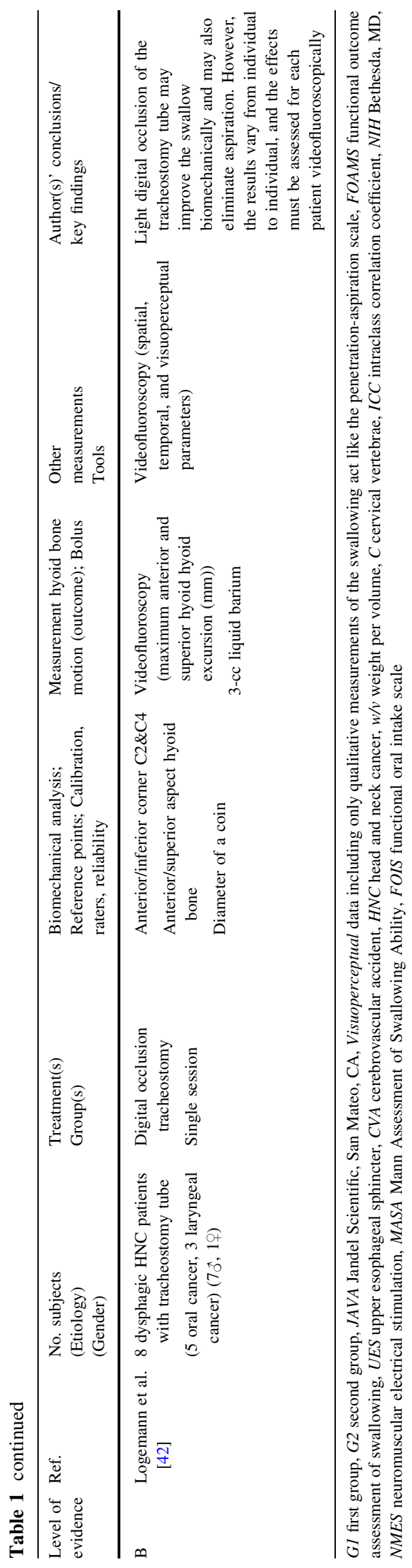

maneuver. None of these maneuvers changed the maximal hyoid bone displacement significantly. A significantly decreased preswallow distance between hyoid and mandible was measured when applying the chin tuck maneuver. Similarly, at preswallow state and during swallowing, a decreased distance between thyroid and hyoid was found using the chin tuck maneuver. The effortful swallow and the chin tuck maneuver resulted in a significant reduction in depth of contrast penetration. None of these techniques resulted in a reduction of misdirected swallows and none influenced the opening of the UES significantly. In this study it was noted that many of the dysphagic subjects had difficulty performing some of the rehabilitation techniques.

Logemann et al. [33] presented data on the effect of the super-supraglottic swallowing maneuver in nine head and neck cancer patients treated with chemoradiation therapy for tumors of the posterior oral cavity. All subjects had severe swallowing problems, as perceived by their physician during or after treatment. After five trials of practicing the maneuver, the dysphagic subjects were analyzed in a single-session design during VFS while performing the maneuver. Overall, fewer swallowing disorders were observed when the patients used the super-supraglottic swallow than when they did not. Five of nine subjects showed significant changes in swallowing during the intake of 1-cc boluses. Maximal hyoid elevation at the time of first cricopharyngeal opening and overall maximal hyoid elevation were increased significantly during the maneuver. Five patients showed significantly increased maximal hyoid elevation with the maneuver during the 3-cc trails. The authors concluded that the super-supraglottic swallow maneuver resulted in improved biomechanics of swallowing in irradiated head and neck cancer patients.

Surface electrical stimulation has been described in the literature as a means to facilitate swallowing. Hypotheses suggested that electrodes for surface electrical stimulation may simultaneously activate the submental and laryngeal regions of the throat, with the aim of producing a simultaneous contraction of the mylohyoid in the submental region (to elevate the hyoid bone) and the thyrohyoid in the neck (to elevate the larynx to the hyoid bone) [44]. The gradually rising intensity of the electrical current (motor stimulation) may cause a depolarization of nerve endings in muscles lying beneath the skin's surface, leading to muscle contraction [24, 45-47].

Ludlow et al. [36] studied the effect of surface electrical stimulation on a small population of chronically dysphagic subjects $(N=11)$. Ten subjects received motor stimulation at rest, ten received motor stimulation swallow trials, and eight received low sensory levels of stimulation. Statistical analysis showed significant inferior hyoid bone displacement during motor stimulation at rest. Posterior hyoid bone displacement on the $x$ axis was not significant during motor 
stimulation at rest. Sensory stimulation levels during swallowing showed significant improvement on the NIH Swallowing Safety Scale (NIH-SSS) but not on the Penetration-Aspiration Scale [48]. Scores on these two scales did not differ significantly between the nonstimulated swallows and the motor-stimulated swallows. A reduction on the Penetration-Aspiration Scale during motor stimulation while swallowing was significantly inversely related to the degree of inferior hyoid bone displacement at rest during motor stimulation. The authors found an unexpected relationship indicating that dysphagic subjects with the greatest hyoid depression during motor stimulation at rest had the greatest improvement during swallowing with the same levels of stimulation.

Many studies evaluated the effect of bolus modification on swallowing. Bolus modification was evaluated as an intervention effect in the three studies described below.

Kendall et al. [35] evaluated swallowing in elderly dysphagic subjects and compared those results with those of younger and older healthy control subjects $(N=65)$. This evaluation was performed using different bolus volumes. The hyoid bone elevation was increased in female dysphagic subjects compared to younger healthy female control subjects during the ingestion of a 1-cc bolus. Three male dysphagic subjects showed decreased hyoid elevation during the ingestion of $20-\mathrm{cc}$ boluses in contrast to the healthy younger and older men. The distance of hyoid bone elevation was usually greater for the larger boluses. The patients elevated the hyoid bone significantly farther than normal during a small-bolus swallow. This was also seen, though to a lesser extent, in the older control group. Temporal measurements in VFS showed delayed hyoid bone displacements in the dysphagic subjects compared to the younger healthy control subjects. The application of 20cc boluses in the dysphagic subjects resulted in significantly earlier onset of superior hyoid bone displacement relative to bolus arrival in the vallecula compared to the younger healthy control subjects. The duration between the initiation of swallowing and the moment of maximal hyoid bone displacement was prolonged in dysphagic subjects during the intake of 20-cc boluses. The duration of the position of maximal hyoid bone displacement was shortened in the patients as well as in the elderly healthy subjects for 1-cc boluses. The authors concluded that an increased extent of hyoid bone displacement in older dysphagic subjects may represent a necessary compensation designed to minimize the effect of the short duration of hyoid elevation on the UES opening.

Kendall et al. [36] performed a preliminary study to identify swallowing characteristics in head and neck cancer patients treated with chemoradiation therapy $(N=20)$. In this study different bolus volumes and consistencies were used in patients than in healthy control subjects. Aspiration was observed in two patients with tongue base tumors while swallowing a small-sized thin liquid bolus. During the application of a paste bolus, aspiration disappeared. In male patients a significant reduction in hyoid bone displacement was demonstrated for all bolus volumes and consistencies compared to healthy male control subjects. The amplitude of hyoid bone displacement did not differ across the tumor location groups. In the healthy control subjects, hyoid bone displacement was influenced by bolus size and by gender. Larynx-to-hyoid approximation was diminished in the patient group. The laryngeal displacement decreased significantly in the radiotherapy patients compared to normal subjects, except for the paste bolus. The amplitude of the UES opening was preserved despite radiotherapy. Increased bolus volume caused increased UES opening. The pharyngeal area at maximal constriction was significantly larger in the radiotherapy patients than in the healthy control subjects, indicating a less effective mechanism of pharynx constriction against the tongue base in the patients. The authors concluded that following radiotherapy, it is likely that even patients with no complaints of dysphagia will demonstrate an abnormal swallow.

In the study of Wintzen et al. [37], 21 Parkinson patients were compared to their spouses during the evaluation of the effects of different bolus volumes on swallowing. The starting position of the hyoid bone in Parkinson patients did not change with increasing bolus volumes. However, in the healthy control subjects, an adaptive descent of the hyoid bone in the starting position was observed using increasing bolus volumes. The mean amplitude of maximal superior hyoid bone displacement did not differ between Parkinson patients and healthy control subjects. The mean amplitude of hyoid bone displacement was also not significantly influenced by bolus size. Another outcome parameter was the number of swallows with hesitancy during the onset of swallowing. This number was significantly higher in the Parkinson group. The authors concluded that the lack of adaptative descent in the rest position of the hyoid bone could be explained by hypokinesia; however, the amplitude of maximal hyoid bone displacement was not influenced by hypokinesia.

Four studies combined different interventions and analyzed their effects on hyoid bone displacement.

A recent study published by Logemann et al. [38] compared the Shaker exercise program to traditional swallowing therapy in dysphagic subjects $(N=14)$. The traditional swallowing therapy involved a series of exercises that included different swallowing maneuvers and several specific well-defined tongue exercises. Postswallow aspiration decreased significantly in the Shaker exercise group compared to the traditional swallowing therapy group. Post therapy, the location of oral and pharyngeal 
residue did not significantly differ between the treatment groups. Following traditional swallowing therapy, the superior hyoid bone displacement and superior laryngeal displacement increased significantly. Following the Shaker exercise program and traditional therapy, the width of the UES opening increased significantly. The authors concluded that traditional swallowing therapy and the Shaker exercise program have different effects. Based on their findings, the authors concluded that after swallowing aspirating patients, in particular, should follow the Shaker exercise program. Patients showing a reduced range of displacement of their pharyngeal structures would preferably be given traditional swallowing therapy.

Carnaby-Mann and Crary [39] presented data from a prospective case series that investigated the influence of neuromuscular electrical stimulation combined with functional swallowing activities in chronically dysphagic subjects $(N=5)$. Four stimulating electrodes, representing two channels, were placed on the anterior neck midline in a vertical arrangement: two electrodes superior and two inferior to the thyroid notch. Subjects practiced during 15 treatment sessions. Post therapy, there was significant improvement in all primary clinical outcome parameters, including the Mann Assessment of Swallowing Ability (MASA) [49], the Functional Oral Intake Scale (FOIS), nutritional status, and the patient's perception of swallowing ability. No long-term changes were observed after the 6-month follow-up. Post therapy, larynx and hyoid showed increased elevation using a 10-cc thick liquid bolus. However, the opposite pattern was observed using a 5-ce thin liquid bolus. The authors concluded that this program of swallowing therapy resulted in clinical and functional gains for chronically dysphagic subjects.

Ciucci et al. [40] described the effects of deep brain stimulation (DBS) and modulation of bolus volume on swallowing in Parkinson patients $(N=14)$. No significant effects were found for the DBS condition on maximal hyoid bone displacement. Furthermore, hyoid bone displacement showed a tendency to occur in the opposite direction to that expected. Results showed a main effect of increasing bolus volume on maximal hyoid bone displacement in all DBS conditions. More impairment occurred in the 10-cc bolus condition compared with the 5-cc condition. The pharyngeal transit time and visuoperceptual analysis of the pharyngeal phase (pharyngeal total composite score, e.g., decreased velar elevation and laryngeal penetration) improved in the DBS ON condition. The authors concluded that the degree of hyoid bone excursion and oral-stage measures did not improve, suggesting that these motor acts may be under the control of different sensorimotor pathways within the basal ganglia.

Suiter et al. [41] analyzed the effect of tracheostomy cuff status and one-way speaking-valve placement using bolus modification in dysphagic subjects $(N=18)$. Cuff status significantly influenced the anterior hyoid bone displacement. A deflated cuff increased the amplitude of anterior hyoid bone displacement. There was also a significant bolus effect resulting in increased maximal anterior hyoid bone displacement using liquid bolus compared to the pureed bolus (cuff-inflated condition versus the oneway speaking-valve condition). However, laryngeal elevation was not significantly influenced by cuff status or one-way speaking-valve placement. The cuff-inflated condition versus the one-way speaking-valve condition showed no significant effects on the amplitude of maximal anterior hyoid bone displacement. Neither penetration nor aspiration was significantly affected by cuff status. However, one-way speaking-valve placement significantly reduced scores on the Penetration-Aspiration scale [48] compared to cuff-inflated and cuff-deflated conditions. The authors concluded that clinicians who complete VFS with tracheostomized patients should include several bolus presentations with a one-way speaking valve in place before making any decisions about using the valve to reduce aspiration.

Logemann et al. [42] examined the effect of digital occlusion of the tracheostomy in eight dysphagic subjects treated for head and neck cancer $(N=6$ surgical treatment, $N=2$ chemoradiotherapy). Lightly digital occlusion resulted in a minor reduction of swallowing disorders. Two of four aspirating subjects showed eliminated aspiration after the swallow during occlusion of the tracheostomy tube. These two patients showed reduced laryngeal elevation without occlusion. Digital occlusion resulted in some significant biomechanical changes. First, both the maximal superior laryngeal elevation and the laryngeal elevation at the time of initial UES opening increased. Second, the hyoid bone elevation at the time of initial UES opening increased. Third, the duration of tongue base contact with the posterior pharyngeal wall was reduced and the onset of anterior displacement of the posterior pharyngeal wall occurred later. The authors concluded that swallowing characteristics showed interindividual variation as well as variation during changes of tracheostomy cuff status. VFS is advised for each patient in order to evaluate these conditions.

\section{Discussion}

Intervention Effects in the Literature

It may be concluded that hyoid bone displacement showed statistically significant intervention effects in many studies with dysphagic subjects. While the Shaker exercise program did not significantly influence hyoid bone 
displacement $[31,38]$, it did significantly improve other biomechanical parameters and reduce postswallow aspiration $[31,38]$. Traditional swallowing exercise and the supersupraglottic swallow showed increased superior hyoid bone displacement [33, 38]. Evaluation of specific maneuvers like the effortful swallow, the supraglottic swallow, and the chin tuck showed no significant intervention effects on hyoid bone displacement [32]. The effortful swallow maneuver and chin tuck applied in dysphagic subjects did show reduction of penetration [32]. Bolus volume and consistency modification resulted in variable effects on hyoid bone displacement in dysphagic subjects. Three studies showed significant effects from bolus modification [36, 40, 41]. In dysphagic subjects with a tracheostomy, the anterior hyoid bone displacement increased significantly with a liquid bolus compared to a pureed bolus [41]. In Parkinson patients, the maximal hyoid bone displacement increased significantly with increased bolus sizes [40]. In healthy control subjects, the same effect of increased superior hyoid bone displacement was seen with increased bolus sizes [36]. Both DBS and NMES showed opposite effects on hyoid bone displacement in dysphagic subjects [34, 40]. During DBS stimulation, the hyoid bone tended to move inferiorly, in contrast to the superior displacement caused by other swallowing interventions. Carnaby-Mann and Crary [39] combined the NMES with functional swallowing activities. These combined interventions showed an increased superior hyoid bone displacement with a 10-cc thick liquid bolus. In contrast, the opposite effect was seen with a 5-cc thin liquid bolus, with less elevation following therapy. The last intervention to be mentioned is the effect of tracheostomy status on hyoid bone displacement. Cuff deflation resulted in significantly increased anterior hyoid bone displacement [41]. During digital occlusion of the tracheostomy, hyoid bone elevation at the time of initial UES opening increased significantly [42]. However, maximal superior and anterior hyoid bone displacement were not significantly influenced by digital occlusion [42].

\section{Methodological Problems}

The systematic search conducted for this review generated a limited number of articles on the biomechanical analysis of hyoid bone displacement in VFS to evaluate intervention effects in dysphagic subjects. Only 2 of the 12 included articles met the level A criteria [31,38]. All studies varied in quality of design, frequently showing diverse methodological shortcomings. The requirements to obtain sufficiently reliable study results are blinding of the raters combined with consensus training and independent scoring. Only six studies described the blinding and reliability analysis of the raters and used more than one rater [31, 34 ,
36, 38-40]. Five studies did not present any information about the raters, resulting in less robust conclusions [32, $33,35,37,42]$.

Study size varied from 5 to 65 subjects. However, most of the studies using dysphagic subjects were rather small. Four studies analyzed a population of fewer than ten subjects $[32,33,39,42]$. Several small-sized populations used parametric statistics, thus assuming a normal distribution; however, no information on the data distribution was presented [34-41]. Five studies used very heterogeneous dysphagic populations and did not deal with possible confounding factors like etiology, age, and gender [31, 32, 34, 38, 39]. Three studies compared dysphagic subjects to healthy control subjects. Kendall et al. [35] described a group of 23 healthy control subjects matched for age and gender with the patients. However, in another study of Kendall et al. [36], 60 healthy control subjects were evaluated without presenting any subject characteristics. Wintzen et al. [37] chose the spouses of the dysphagic subjects as healthy control subjects, not matched for gender. In some articles intervention effects were analyzed during the immediate phase following, for example, stroke, surgery, or radiotherapy, not taking spontaneous recovery during therapy into account $[31-33,36]$.

When analyzing hyoid bone displacement, different software applications can be used. Investigators should describe the applied reference points on the hyoid bone and on structures serving as a coordinate system ( $x, y$ axes). Only 4 of the 12 studies described the applied reference points [34, 39, 40, 42]. Finally, few studies used more than one assessment tool to evaluate the intervention effects [31, 32, 39].

In conclusion, because of the different study designs, small-sized heterogeneous populations, different ways of evaluating hyoid bone displacement, and methodological shortcomings, the results of these studies could not be compared with one another using statistical pooling.

\section{Future Research}

This article presents a review of the evaluation of intervention effects on swallowing, using biomechanical analysis of hyoid bone displacement in VFS as the outcome parameter. This review has shown that VFS analysis of the spatial outcome parameter hyoid bone displacement is able to detect significant intervention effects. For future research, it is nonetheless advisable to use well-defined subject populations and well-defined videofluoroscopic protocols, including the description and application of the software program used for tracking hyoid bone displacement. Furthermore, future studies should be based on an adequate number of subjects, more than one assessment 
tool, and well-defined outcome parameters. The challenge that lies ahead is to develop a universal standardized software application to analyze hyoid bone displacement, which would make it easier to compare the studies with one another.

Open Access This article is distributed under the terms of the Creative Commons Attribution Noncommercial License which permits any noncommercial use, distribution, and reproduction in any medium, provided the original author(s) and source are credited.

\section{References}

1. Logemann JA, Pauloski BR, Rademaker AW, Kahrilas PJ. Oropharyngeal swallow in younger and older women: videofluoroscopic analysis. J Speech Lang Hear Res. 2002;45:434-45.

2. Logemann JA, Pauloski BR, Rademaker AW, Colangelo LA, Kahrilas PJ, Smith CH. Temporal and biomechanical characteristics of oropharyngeal swallow in younger and older men. J Speech Lang Hear Res. 2000;43:1264-74.

3. Cook IJ. Oropharyngeal dysphagia. Gastroenterol Clin North Am. 2009;38:411-31.

4. Suarez-Cunqueiro MM, Schramm A, Schoen R, Seoane-Leston J, Otero-Cepeda XL, Bormann KH, Kokemueller H, Metzger, DizDios P, Gellrich NC. Speech and swallowing impairment after treatment for oral and oropharyngeal cancer. Arch Otolaryngol Head Neck Surg 2008;134:1299-1304

5. Sue Eisenstadt E. Dysphagia and aspiration pneumonia in older adults. J Am Acad Nurse Pract. 2010;22:17-22.

6. Perry L. Dysphagia: the management and detection of a disabling problem. Br J Nurs. 2001;10:837-44.

7. Kellen PM, Becker DL, Reinhardt JM, Van Daele DJ. Computerassisted assessment of hyoid bone motion from videofluoroscopic swallow studies. Dysphagia. 2010;25(4):298-306.

8. Logemann JA. Swallowing disorders. Best Pract Res Clin Gastroenterol. 2007;21:563-73.

9. Nakane A, Tohara H, Ouchi Y, Goto S, Uematsu H. Videofluoroscopic kinesiologic analysis of swallowing: defining a standard plane. J Med Dent Sci. 2006;53:7-15.

10. Palmer JB, Kuhlemeier KV, Tippett DC, Lynch C. A protocol for the videofluorographic swallowing study. Dysphagia. 1993;8: 209-14.

11. Dengel G, Robbins J, Rosenbek JC. Image processing in swallowing and speech research. Dysphagia. 1991;6:30-9.

12. Logemann JA, Kahrilas PJ, Begelman J, Dodds WJ, Pauloski BR. Interactive computer program for biomechanical analysis of videoradiographic studies of swallowing. AJR Am J Roentgenol. 1989;153:277-80.

13. Perlman AL, VanDaele DJ, Otterbacher MS. Quantitative assessment of hyoid bone displacement from video images during swallowing. J Speech Hear Res. 1995;38:579-85.

14. Dodds WJ, Stewart ET, Logemann JA. Physiology and radiology of the normal oral and pharyngeal phases of swallowing. AJR Am J Roentgenol. 1990;154:953-63.

15. Dodds WJ, Logemann JA, Stewart ET. Radiologic assessment of abnormal oral and pharyngeal phases of swallowing. AJR Am J Roentgenol. 1990;154:965-74.

16. Dodds WJ, Man KM, Cook IJ, Kahrilas PJ, Stewart ET, Kern MK. Influence of bolus volume on swallow-induced hyoid movement in normal subjects. AJR Am J Roentgenol. 1988;150:130-1307.

17. Paik NJ, Kim SJ, Lee HJ, Jeon JY, Lim JY, Han TR. Movement of the hyoid bone and the epiglottis during swallowing in patients with dysphagia from different etiologies. J Electromyogr Kinesiol. 2008;18:329-35.

18. Easterling CS, Bousamra M 2nd, Lang IM, Kern MK, Nitschke T, Bardan E, Shaker R. Pharyngeal dysphagia in postesophagectomy patients: correlation with deglutitive biomechanics. Ann Thorac Surg. 2000;69:989-92.

19. Lazarus CL, Logemann JA, Pauloski BR, Colangelo LA, Kahrilas PJ, Mittal BB, Pierce M. Swallowing disorders in head and neck cancer patients treated with radiotherapy and adjuvant chemotherapy. Laryngoscope. 1996;106:1157-66.

20. Martin RE, Letsos P, Taves DH, Inculet RI, Johnston H, Preiksaitis HG. Oropharyngeal dysphagia in esophageal cancer before and after transhiatal esophagectomy. Dysphagia. 2001;16:23-31.

21. Terk AR, Leder SB, Burrell MI. Hyoid bone and laryngeal movement dependent upon presence of a tracheotomy tube. Dysphagia. 2007;22:89-93.

22. Yuceturk AV, Tarhan S, Gunhan K, Pabuscu Y. Videofluoroscopic evaluation of the swallowing function after supracricoid laryngectomy. Eur Arch Otorhinolaryngol. 2005;262:198-203.

23. Lazarus C, Logemann JA, Gibbons P. Effects of maneuvers on swallowing function in a dysphagic oral cancer patient. Head Neck. 1993;15:419-24.

24. Park CL, O'Neill PA, Martin DF. A pilot exploratory study of oral electrical stimulation on swallow function following stroke: an innovative technique. Dysphagia. 1997;12:161-6.

25. Roos G, Leysen H, Van Wassenbergh S, Herrel A, Jacobs P, Dierick M, Aerts P, Adriaens D. Linking morphology and motion: a test of a four-bar mechanism in seahorses. Physiol Biochem Zool. 2009;82:7-19.

26. Kendall KA, McKenzie SW, Leonard RJ, Jones CU. Timing of swallowing events after single-modality treatment of head and neck carcinomas with radiotherapy. Ann Otol Rhinol Laryngol. 2000;109:767-75.

27. Leonard R, McKenzie S. Hyoid-bolus transit latencies in normal swallow. Dysphagia. 2006;21:183-90.

28. Delmas A, Senecail B. [Biometric aspects of hyoid mechanics in man]. Bull Assoc Anat (Nancy). 1977;61:189-98.

29. Huckabee ML, Butler SG, Barclay M, Jit S. Submental surface electromyographic measurement and pharyngeal pressures during normal and effortful swallowing. Arch Phys Med Rehabil. 2005;86:2144-9.

30. Siwek J, Gourlay ML, Slawson DC, Shaughnessy AF. How to write an evidence-based clinical review article. Am Fam Physician. 2002;65:251-8.

31. Shaker R, Easterling C, Kern M, Nitschke T, Massey B, Daniels S, Grande B, Kazandjian M, Dikeman K. Rehabilitation of swallowing by exercise in tube-fed patients with pharyngeal dysphagia secondary to abnormal UES opening. Gastroenterology. 2002;122:1314-21.

32. Bülow M, Olsson R, Ekberg O. Videomanometric analysis of supraglottic swallow, effortful swallow, and chin tuck in patients with pharyngeal dysfunction. Dysphagia. 2001;16:190-5.

33. Logemann JA, Pauloski BR, Rademaker AW, Colangelo LA. Super-supraglottic swallow in irradiated head and neck cancer patients. Head Neck. 1997;19:535-40.

34. Ludlow CL, Humbert I, Saxon K, Poletto C, Sonies B, Crujido L. Effects of surface electrical stimulation both at rest and during swallowing in chronic pharyngeal dysphagia. Dysphagia. 2007;22:1-10.

35. Kendall KA, Leonard RJ. Hyoid movement during swallowing in older patients with dysphagia. Arch Otolaryngol Head Neck Surg. 2001;127:1224-9.

36. Kendall KA, McKenzie SW, Leonard RJ, Jones C. Structural mobility in deglutition after single modality treatment of head and neck carcinomas with radiotherapy. Head Neck. 1998;20:720-5. 
37. Wintzen AR, Badrising UA, Roos RA, Vielvoye J, Liauw L. Influence of bolus volume on hyoid movements in normal individuals and patients with Parkinson's disease. Can J Neurol Sci. 1994;21:57-9.

38. Logemann JA, Rademaker A, Pauloski BR, Kelly A, StanglMcBreen C, Antinoja J, Grande B, Farquharson J, Kern M, Easterling C, Shaker R. A randomized study comparing the Shaker exercise with traditional therapy: a preliminary study. Dysphagia. 2009;24:403-11.

39. Carnaby-Mann GD, Crary MA. Adjunctive neuromuscular electrical stimulation for treatment-refractory dysphagia. Ann Otol Rhinol Laryngol. 2008;117:279-87.

40. Ciucci MR, Barkmeier-Kraemer JM, Sherman SJ. Subthalamic nucleus deep brain stimulation improves deglutition in Parkinson's disease. Mov Disord. 2008;23:676-83.

41. Suiter DM, McCullough GH, Powell PW. Effects of cuff deflation and one-way tracheostomy speaking valve placement on swallow physiology. Dysphagia. 2003;18:284-92.

42. Logemann JA, Pauloski BR, Colangelo L. Light digital occlusion of the tracheostomy tube: a pilot study of effects on aspiration and biomechanics of the swallow. Head Neck. 1998;20:52-7.

43. Logemann J. Evaluation and treatment of swallowing disorders. 2nd ed. Austin, TX: Pro-Ed, Inc; 1998.

44. Freed M, Wijting Y. VitalStim Certification Program. Training manual for patient assessment and treatment using VitalStim electrical stimulation. Hixson TN: Chattanooga Group; 2003.
45. Freed ML, Freed L, Chatburn RL, Christian M. Electrical stimulation for swallowing disorders caused by stroke. Respir Care. 2001;46:466-74.

46. Leelamanit V, Limsakul C, Geater A. Synchronized electrical stimulation in treating pharyngeal dysphagia. Laryngoscope. 2002;112:2204-10.

47. Power M, Fraser C, Hobson A, Rothwell JC, Mistry S, Nicholson DA, Thompson DG, Hamdy S. Changes in pharyngeal corticobulbar excitability and swallowing behavior after oral stimulation. Am J Physiol Gastrointest Liver Physiol. 2004;286:G45-50.

48. Rosenbek JC, Robbins JA, Roecker EB, Coyle JL, Wood JL. A penetration-aspiration scale. Dysphagia. 1996;11:93-8.

49. Mann G. The Mann Assessment of Swallowing Ability. Clifton Park, NY: Singular Thompson Learning; 2001.

Jolien G. J. van der Kruis MD

Laura W. J. Baijens MD

Renée Speyer MS

Iris Zwijnenberg MD 